\title{
Brain metastases, patterns of intracranial progression, and the clinical value of upfront cranial radiotherapy in patients with metastatic non-small cell lung cancer treated with PD-1/PD-L1 inhibitors
}

Tiantian Guo ${ }^{1,2}$, Li Chu ${ }^{1,2}$, Xiao Chu ${ }^{1,2}$, Xi Yang ${ }^{1,2}$, Yida $\mathrm{Li}^{1,2}$, Yue Zhou ${ }^{1,2}$, Dayu $\mathrm{Xu}^{1,2}$, Jinmeng Zhang ${ }^{1,2}$, Shengping Wang ${ }^{2,3}$, Jie $\mathrm{Hu}^{4}$, Qian Chu ${ }^{5}$, Teresa Moran ${ }^{6,7}$, William Chi-Shing Cho ${ }^{8}$, Kenneth W. Merrell', Stefania Rizzo ${ }^{10}$, Yanfei Liu ${ }^{2,11}$, Jianjiao $\mathrm{Ni}^{1,2}$, Zhengfei Zhu ${ }^{1,2,12}$

${ }^{1}$ Department of Radiation Oncology, Fudan University Shanghai Cancer Center, Shanghai, China; ${ }^{2}$ Department of Oncology, Shanghai Medical College, Fudan University, Shanghai, China; ${ }^{3}$ Department of Radiology, Fudan University Shanghai Cancer Center, Shanghai, China; ${ }^{4}$ Department of Pulmonary Medicine, Zhongshan Hospital, Fudan University, Shanghai, China; ${ }^{5}$ Department of Oncology, Tongji Hospital, Tongji Medical College, Huazhong University of Science and Technology, Wuhan, China; ${ }^{6}$ Medical Oncology Department, Catalan Institute of Oncology, Hospital Universitari Germans Trias i Pujol, Badalona Applied Research Group in Oncology, Badalona, Spain; ${ }^{7}$ Department of Medicine, Universitat Autonoma de Barcelona, Badalona, Spain; ${ }^{8}$ Department of Clinical Oncology, Queen Elizabeth Hospital, Hong Kong, China; ${ }^{9}$ Department of Radiation Oncology, Mayo Clinic, Rochester, MN, USA; ${ }^{10}$ Imaging Institute of the Southern Switzerland (IIMSI), Ente Ospedaliero Cantonale (EOC), Università della Svizzera italiana, Lugano, Switzerland; ${ }^{11}$ Office of Clinical Research, Fudan University Shanghai Cancer Center, Shanghai, China; ${ }^{12}$ Institute of Thoracic Oncology, Fudan University, Shanghai, China

Contributions: (I) Conception and design: Z Zhu, J Ni, Y Liu, T Guo; (II) Administrative support: Z Zhu, J Ni, Y Liu; (III) Provision of study materials or patients: L Chu, X Yang, X Chu, J Hu, Q Chu; (IV) Collection and assembly of data: T Guo, L Chu, X Chu, X Yang, Y Li, Y Zhou, D Xu, J Zhang, S Wang, J Hu, Q Chu; (V) Data analysis and interpretation: T Guo; (VI) Manuscript writing: All authors; (VII) Final approval of manuscript: All authors.

Correspondence to: Yanfei Liu, MN. Office of Clinical Research, Fudan University Shanghai Cancer Center, 270 Dong An Road, Shanghai, China. Email: liuyanfei@fudan.edu.cn; Jianjiao Ni, MD. Department of Radiation Oncology, Fudan University Shanghai Cancer Center, 270 Dong An Road, Shanghai, China. Email: nijianjiao8@sina.com; Zhengfei Zhu, MD, PhD. Department of Radiation Oncology, Fudan University Shanghai Cancer Center, 270 Dong An Road, Shanghai, China. Email: fuscczzf@163.com.

Background: Despite the emergence of programmed death 1/programmed death-ligand 1 (PD-1/PD-L1) inhibitors in the treatment of non-small cell lung cancer (NSCLC) patients with brain metastases (BMs), knowledge gaps remain regarding the impact and timing of cranial radiotherapy for patients receiving antiPD-1/PD-L1 therapy.

Methods: Data were collected from 461 consecutive patients who received anti-PD-1/PD-L1 therapy for metastatic NSCLC at three institutions between June 2017 and September 2020. Intracranial progressive disease (PD) at the original disease sites, new sites, or both sites were classified as original-site PD (OPD), new-site PD (NPD), and original-and-new-site PD (ONPD), respectively. Patients with baseline BMs were categorized based on whether they received upfront cranial radiotherapy (uCRT) at any time point between the introduction of anti-PD-1/PD-L1 therapy and the first subsequent progression.

Results: Of the 461 patients enrolled, 110 (23.9\%) had BMs at baseline. The presence of BMs did not show independent prognostic value for progression-free survival (PFS) or overall survival (OS). During a median follow-up of 13.2 months, 96 patients with BMs developed PD, of whom 53 (55.2\%) experienced intracranial PD. OPD, NPD, and ONPD were observed in 50.9\%, $18.9 \%$, and $30.2 \%$ of patients, respectively. Patients who received uCRT exhibited a longer median OS than those with BMs who did not receive uCRT (25.4 vs. 14.6 months, HR: 0.52, 95\% CI: 0.29-0.91, $\mathrm{P}=0.041$ ); this survival advantage was more prominent in patients with 1-4 BMs (median OS, 25.4 vs. 17.0 months, HR: 0.42, 95\% CI: $0.22-0.81, \mathrm{P}=0.024$ ), and uCRT was independently associated with OS among these patients. 


\begin{abstract}
Conclusions: The presence of BMs at baseline was not associated with poorer OS in patients with metastatic NSCLC treated with anti-PD-1/PD-L1 therapy. Intracranial progression on PD-1/PD-L1 inhibitors predominately occurred at the original BM sites. The use of uCRT may improve OS, especially in NSCLC patients with 1-4 BMs.
\end{abstract}

Keywords: Cranial radiotherapy; brain metastases (BMs); immune checkpoint inhibitors; non-small cell lung cancer (NSCLC)

Submitted Jun 24, 2021. Accepted for publication Feb 11, 2022.

doi: $10.21037 /$ tlcr-22-54

View this article at: https://dx.doi.org/10.21037/tlcr-22-54

\section{Introduction}

Historically, the dismal outcomes of patients with non-small cell lung cancer (NSCLC) with brain metastases (BMs) have often led to considerable therapeutic nihilism. However, evolving radiotherapy (RT) techniques, together with the advances in neuroimaging and medical oncology developed in the past few decades, have dramatically transformed the therapeutic landscape of brain-metastatic NSCLC. In particular, the unique pharmacokinetics/pharmacodynamics of programmed death receptor (ligand) 1 (PD-1/PD-L1) inhibitors are challenging the traditional notion of the brain as a fully protected biological sanctuary (1). For example, in high-grade gliomas, cerebrospinal fluid (CSF) concentrations of systemically delivered pembrolizumab show a mean CSF:serum ratio of 0.009 , and CSF pembrolizumab concentrations were sufficient to block PD-1 in functional assays, suggesting that systemically administered PD-1/ PD-L1 inhibitors could elicit a local effect in the central nervous system (CNS) (2). Unfortunately, most pivotal trials of PD-1/PD-L1 inhibitors in advanced NSCLC have excluded patients with untreated and/or active BMs (3-6). Consequently, knowledge gaps remain regarding the role of PD-1/PD-L1 inhibitors in the treatment of brain-metastatic NSCLC, and data from real-world clinical practice with less-selected populations are therefore urgently needed.

Given the importance of RT as the mainstay of local treatment for patients with $\mathrm{BMs}$, the relative roles of cranial RT and anti-PD-1/PD-L1 therapy are of considerable clinical interest for defining the optimal treatment strategy for brain-metastatic NSCLC. A growing body of literature suggests that the combination of RT and PD-1/PD-L1 inhibitors could be superior to RT alone or exclusively systemic therapy in terms of local control and survival (7-10). However, this combination strategy has not been fully optimized and some questions remain unanswered, such as the appropriate treatment sequences.

Herein, we hypothesize that upfront cranial radiotherapy (uCRT) delivered before disease progression on PD-1/PDL1 inhibitors may provide better local and distant tumor control or even improve overall survival (OS) compared to PD-1/PD-L1 inhibitors alone. This hypothesis is based on the idea that immune checkpoint inhibitor-resistant clones which lack particular tumor antigens or antigen presentation in persistent intracranial lesions can serve as seeds for new metastases and lead to distant brain failure. Moreover, cranial irradiation is more likely to achieve potent antitumor efficacy in patients with a lower CNS disease burden than in patients with progressive intracranial disease after anti-PD-1/PD-L1 therapy. However, there is a paucity of data available regarding the patterns of initial and intracranial progression on PD-1/PD-L1 inhibitors, and the efficacy of uCRT in patients with PD-1/PD-L1 inhibitortreated brain-metastatic NSCLC stratified by the number of BMs has not been explored in a large patient population.

Therefore, we performed a retrospective multiinstitutional study to examine the impact of the presence of BMs on OS in patients with metastatic NSCLC treated using PD-1/PD-L1 inhibitors. In addition, we present patterns of initial and intracranial progression on PD-1/PDL1 inhibitors and the outcomes of patients with PD-1/PDL1 inhibitor-treated brain-metastatic NSCLC according to the receipt of uCRT (yes $v s$. no) and number of BMs (1-4 $v s$. >4). To limit potential bias in patient selection and data analysis, all consecutive patients with metastatic NSCLC who received $\mathrm{PD}-(\mathrm{L}) 1$ inhibitor-containing treatment regimen at three participating institutions between June 2017 and September 2020 were considered. Multivariate analysis was performed by incorporating treatment modality and baseline characteristics. 
We present the following article in accordance with the STROBE reporting checklist (available at https://tlcr. amegroups.com/article/view/10.21037/tlcr-22-54/rc).

\section{Methods}

\section{Study population and data collection}

A retrospective analysis of patients with metastatic NSCLC at diagnosis or early-stage NSCLC with a recurrence or progression who received immune checkpoint inhibitors of the PD-1/PD-L1-axis (pembrolizumab, nivolumab, camrelizumab, or atezolizumab) monotherapy or combined with other regimens in the metastatic setting at three institutions (Tongji Hospital of Tongji Medical College, Fudan University Zhongshan Hospital, and Fudan University Shanghai Cancer Center) between June 2017 and September 2020 was conducted. All included patients had histologically confirmed NSCLC and complete follow-up data. Patients with leptomeningeal metastases were excluded due to their extremely poor prognosis (11). Electronic patient records were reviewed, and the relevant demographic and clinical information was extracted, including sex, age at anti-PD-1/PD-L1 therapy, line of PD(L)1 inhibitor treatment, smoking history, tumor histology, PD-L1 expression status, Eastern Cooperative Oncology Group performance status (ECOG PS), number of organs harboring metastases, presence or absence of BMs, number of BMs, diameter of the largest BM, clinical presentation (symptomatic $v s$. asymptomatic), treatment regimen (monotherapy or combined with chemotherapy), and prior RT for BMs. Patients with PD-L1 expression $\geq 1 \%$ of tumor cells were defined as PD-L1-positive, which is consistent with the threshold for positive PD-L1 expression used in previous clinical trials evaluating the efficacy of $\mathrm{PD}-(\mathrm{L}) 1$ inhibitors in patients with NSCLC (12).

Follow-up radiological imaging with CT or MRI was typically performed at intervals of $6-8$ weeks. To assess tumor response to therapy, two senior radiologists subjected serial scans to review independently according to the Response Evaluation Criteria in Solid Tumors (RECIST) version 1.1. Available follow-up brain MRI scans were evaluated for intracranial therapeutic response and disease progression at original or new sites in the brain. Intracranial progressive disease $(\mathrm{PD})$ at the original disease sites, new sites, or both were classified as original-site PD (OPD), new-site PD (NPD), and original-and-new-site PD (ONPD), respectively. Data collection was cut-off by March
30,2021 . The study was conducted in accordance with the Declaration of Helsinki (as revised in 2013). The study was approved by the Institutional Review Board of Fudan University Shanghai Cancer Center (No. 2012228-3), Ethics Committee of Zhongshan Hospital, Fudan University (No. B2021-125R), and Ethics Committee of the Tongji Medical College of Huazhong University of Science and Technology (No. TJ-IRB20200731), and individual consent for this retrospective analysis was waived.

\section{$R T$}

The patients with BMs were categorized into two groups (uCRT $v s$. non-uCRT) accordingly to whether they received uCRT at any point between the initiation of anti-PD-1/ PD-L1 therapy and the first subsequent progression event. Whole-brain radiotherapy (WBRT) was delivered at a median dose of 30 Gy (range, 30-40 Gy) in 10-20 fractions. According to each center's clinical practice, single-fraction stereotactic radiosurgery (SRS)/fractionated stereotactic radiotherapy (SRT) schedules ranged from 15-24 Gy in 1 fraction, 20-30 Gy in 3 fractions, 24-32 Gy in 4 fractions, and 25-35 Gy in 5 fractions. The dose fractionation schedules prescribed varied with tumor location, tumor size, and clinical presentation (symptomatic vs. asymptomatic).

\section{Statistical analysis}

Patients' clinical and treatment characteristics were compared using the $\chi^{2}$ test or Fisher's exact test for categorical variables, and unpaired Student's $t$-test or Mann-Whitney $U$ test for continuous variables, as appropriate. Progression-free survival (PFS) was defined as the time from the start of anti-PD-1/PD-L1 therapy to disease progression or death. Intracranial PFS (iPFS) was defined as the time from the start of anti-PD-1/ PD-L1 therapy to radiologically confirmed intracranial progression or death. OS was defined as the time from the start of anti-PD-1/PD-L1 therapy to death. The KaplanMeier method was adopted to estimate PFS, iPFS, and OS, and survival curves were compared using log-rank testing. Cox proportional-hazards regression was used to estimate univariate and multivariate hazard ratios (HRs) and 95\% confidence intervals (CIs) for PFS and OS. Variables were selected for the multivariate analysis based on their statistical significance $(\mathrm{P}<0.10)$ by univariate analysis. Time to intracranial progression was measured from the start of anti-PD-1/PD-L1 therapy to radiologically confirmed 
intracranial progression (event of interest). Competing risk methodology was adopted to perform actuarial univariate and multivariate analyses of factors potentially associated with time to intracranial progression, in which death without the event was defined as a competing risk. A twosided $\mathrm{P}$ value $<0.05$ was considered statistically significant. All statistical analyses were performed with SPSS 21.0 (SPSS, Chicago, IL, USA) and GraphPad Prism 7.0 (La Jolla, CA).

\section{Results}

\section{Patient characteristics}

In total, 461 consecutive patients with metastatic NSCLC who met the eligible criteria were identified from the 3 participating centers (Figure S1). PD-(L)1 inhibitor was received in the first-, second-, and third- or laterline setting in 206 (44.7\%), 138 (29.9\%), and 117 (25.4\%) patients, respectively. 110 (23.9\%) had BMs at the initiation of anti-PD-1/PD-L1 therapy. Table 1 outlines the baseline characteristics of patients with and without BMs. Clinical characteristics were generally comparable between the two groups of patients, except for the BM group being slightly younger (median age, 60 vs. 61 years, $\mathrm{P}=0.045$ ) and having a greater proportion of cases with adenocarcinoma histology [84.5\% (93/110) vs. 61.8\% (217/351), $\mathrm{P}<0.001]$. Among the patients with BMs at baseline, the median number of BMs was 2, and $22(20.0 \%)$ had symptomatic BMs at the initiation of anti-PD-1/PD-L1 therapy. Further details of patients' BMs and treatment characteristics are summarized in Table S1.

\section{Efficacy of anti-PD-1/PD-L1 therapy}

The median follow-up time for the entire cohort is 13.2 months (range, 0.8-44.4 months). There were no significant differences in treatment outcomes among centers. Patients with and without BMs showed an overall response rate (RR) of $24.5 \%$ and $28.2 \%$, respectively $(\mathrm{P}=0.452)$. Among patients that were evaluable for CNS objective response (at least one measurable BM lesion on baseline brain scan according to the RECIST 1.1 criteria) ( $\mathrm{n}=75)$, the intracranial $\mathrm{RR}$ was $28.0 \%$ ( $\mathrm{n}=1$ with complete response and $\mathrm{n}=20$ with partial response). A discordant intracranial and systemic response was observed in 5 patients, including 3 who developed PD in the brain while showing a systemic response, and 2 patients who experienced intracranial response with concurrent rapid systemic progression. Information regarding PD-L1 status was available for 20 of the 75 patients who were evaluable for intracranial outcomes: $12(60 \%)$ patients had PD-L1 positivity (PD-L1 expressed on $\geq 1 \%$ of tumor cells). The intracranial RR was $33.3 \%(4 / 12)$ and $12.5 \%(1 / 8)$ for PD-L1-positive and PD-L1-negative patients, respectively. The median PFS and OS were 3.0 (95\% CI: 2.4-3.6) months and 21.1 (95\% CI: 15.1-27.1) months, respectively, in patients with baseline BMs, and $4.8(95 \%$ CI: 4.2-5.4) months and 20.8 (95\% CI: 17.9-23.7) months, respectively, in patients without baseline BMs (Figure 1). The presence of BMs showed no prognostic association with PFS or OS (Tables S2,S3).

\section{Initial and intracranial progression}

At data cutoff, PD had occurred in 277 (78.9\%) of 351 patients without BMs and $96(87.3 \%)$ of 110 patients with BMs. Patterns of initial progression are shown in Figure 2.

Among the 277 patients without BMs at baseline who progressed, the brain was the first site of progression in $21(7.6 \%)$ patients, including 8 (2.9\%) patients who had intracranial progression detected synchronously with extracranial recurrence. Of the 21 patients with intracranial PD, $10(47.6 \%)$ developed multiple ( $>4)$ brain lesions, whereas the remainder had limited [1-4] BMs. The cumulative actuarial rates of developing BMs at 6,12 , and 18 months were $5.7 \%, 10.0 \%$, and $14.4 \%$, respectively. Univariate analysis uncovered primary tumor size (HR: 6.39, 95\% CI: $1.88-21.71, \mathrm{P}=0.003)$ and the number of organs harboring metastases (HR: 5.60, 95\% CI: 1.88-16.67, $\mathrm{P}=0.002)$ as risk factors associated with the development of BMs. On multivariate analysis, primary tumor size remained significant as a risk factor for BMs (HR: 5.79, 95\% CI: $1.70-19.69, \mathrm{P}=0.005)$, as did the number of organs harboring metastases (HR: 5.07, 95\% CI: 1.70-15.10, $\mathrm{P}=0.004$; Table S4).

Among the 96 patients with baseline BMs who progressed, the brain was the first site of progression in $53(55.2 \%)$ patients, including 31 (32.3\%) patients who had intracranial PD detected synchronously with extracranial progression. OPD, NPD, and ONPD were documented in $27(50.9 \%), 10(18.9 \%)$, and $16(30.2 \%)$ patients, respectively. Of note, in the subgroup of patients who did not receive any local therapy for BMs before disease progression and experienced intracranial $\mathrm{PD}$ by the time of data cutoff $(\mathrm{n}=53), 31(58.5 \%)$ had intracranial PD as the first site of progression, and of them, $16(51.6 \%)$ 
Table 1 Baseline characteristics of patients with advanced NSCLC, stratified according to the presence of BMs

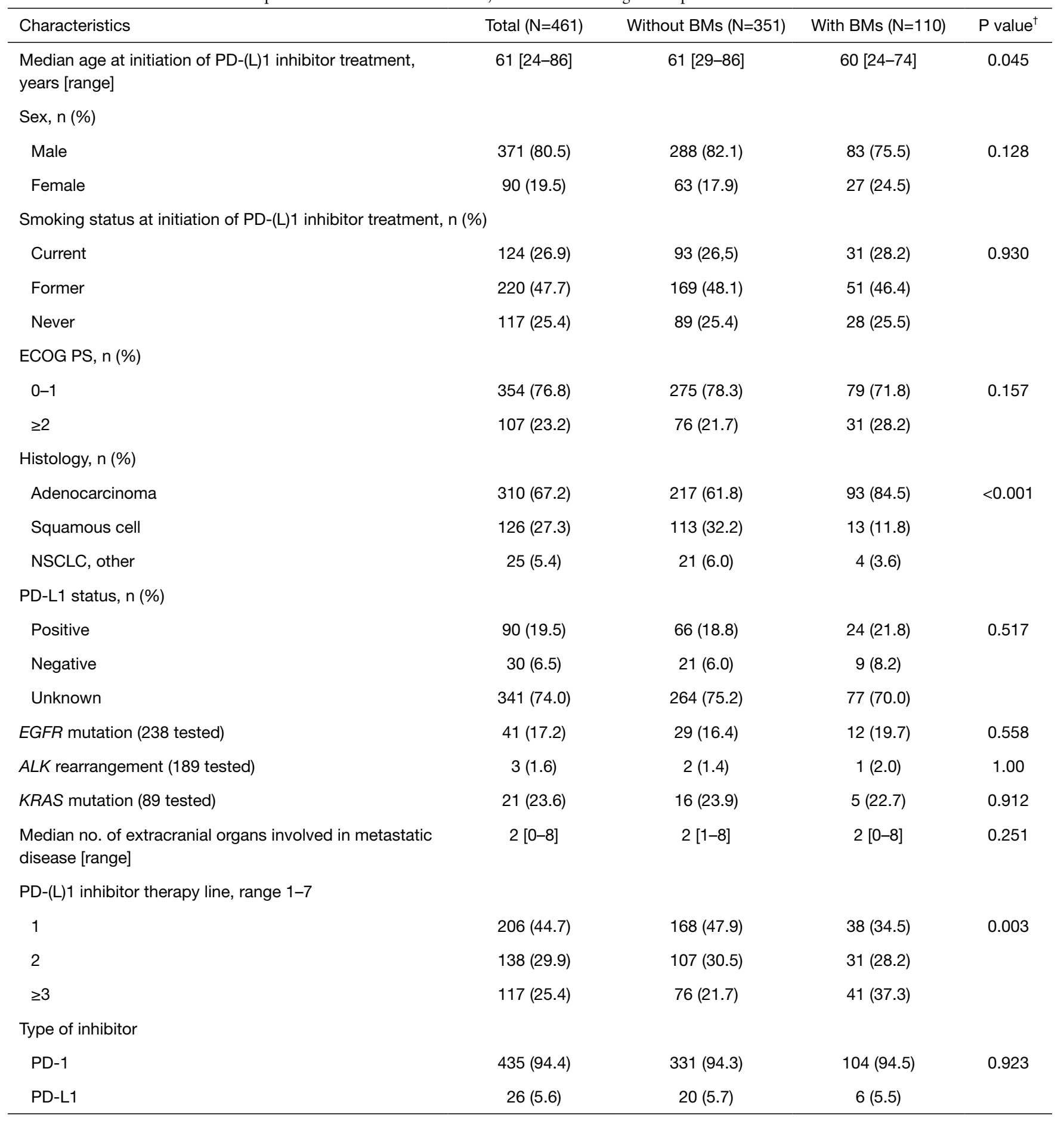

Table 1 (continued) 
Table 1 (continued)

\begin{tabular}{|c|c|c|c|c|}
\hline Characteristics & Total $(\mathrm{N}=461)$ & Without BMs $(\mathrm{N}=351)$ & With BMs (N=110) & $P$ value ${ }^{\dagger}$ \\
\hline \multicolumn{5}{|c|}{ Anti-PD-1/PD-L1 monotherapy } \\
\hline $1^{\text {st }}$ line & $54(11.7)$ & $44(12.5)$ & $10(9.1)$ & 0.077 \\
\hline$\geq 2^{\text {nd }}$ line & $145(31.5)$ & $101(28.8)$ & $44(40.0)$ & \\
\hline $1^{\text {st }}$ line & $152(33.0)$ & $124(35.3)$ & $28(25.5)$ & \\
\hline$\geq 2^{\text {nd }}$ line & $110(23.9)$ & $82(23.4)$ & $28(25.5)$ & \\
\hline
\end{tabular}

${ }^{\dagger}$, patients with and without baseline brain metastases are compared; *, combination of anti-PD-1/PD-L1 therapy and chemotherapy or anti-PD-1/PD-L1 therapy and EGFR-TKIs. ALK, anaplastic lymphoma kinase; NSCLC, non-small cell lung cancer; BMs, brain metastases; ECOG PS, Eastern Cooperative Oncology Group performance status; EGFR, endothelial growth factor receptor; KRAS, Kirsten rat sarcoma; PD-(L)1, programmed death receptor (ligand) 1.
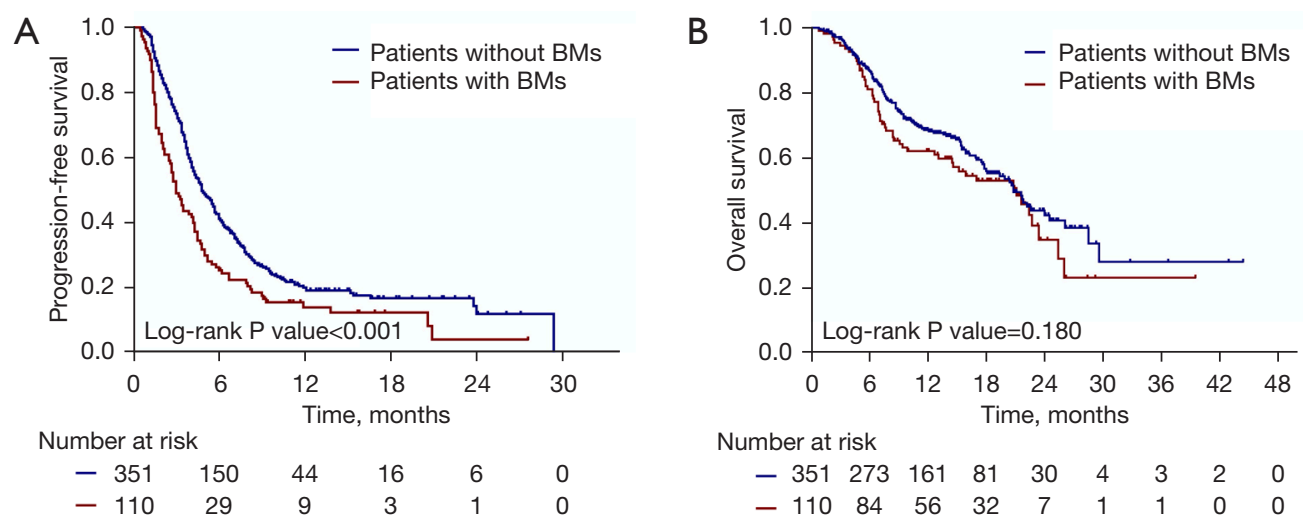

Figure 1 Kaplan-Meier estimates of PFS (A) and OS (B) for patients with baseline BMs versus those without baseline BMs. PFS, progression-free survival; OS, overall survival; BMs, brain metastases.

experienced progressive intracranial disease only at the original BM sites, suggesting a potential role of $\mathrm{uCRT}$ in the treatment of these patients.

\section{Outcomes according to the receipt of $u C R T$ and number of $B M s$}

Of the 110 patients with BMs at baseline, 26 (23.6\%) received uCRT (WBRT: 6, 23.1\%; SRT: 12, 46.2\%; SRS: $8,30.8 \%)$. All patients with $1-4$ BMs $(n=18)$ in the uCRT group received SRS/SRT. For patients with $>4$ BMs who were treated with uCRT $(\mathrm{n}=8), 6(75 \%)$ received WBRT and $2(25 \%)$ received SRS/SRT. The median interval between the initiation of PD-1/PD-L1 inhibitor treatment and the initiation of uCRT was 15 days (inter-quartile range, 13-47 days). The baseline characteristics of patients treated with and without uCRT are summarized in Table 2. Compared with the non-uCRT group, the uCRT group had higher proportions of patients with symptomatic BMs [34.6\% (9/26) vs. $15.5 \%$ (13/84), $\mathrm{P}=0.033$ ] and CNS lesions with a maximum diameter $\geq 10 \mathrm{~mm}[88.5 \%(23 / 26)$ vs. $65.5 \%(55 / 84), \mathrm{P}=0.024]$. Patients treated with uCRT had numerically longer iPFS (median iPFS, 9.3 vs. 4.2 months, HR: $0.58,95 \%$ CI: $0.34-1.02, \mathrm{P}=0.061$, Figure $3 A$ ) and statistically significantly improved PFS and OS compared to those with BMs who did not receive uCRT (median PFS, 4.3 vs. 2.7 months, HR: 0.62 , 95\% CI: $0.40-0.95, \mathrm{P}=0.043$, Figure 3B; median OS, 25.4 vs. 14.6 months, HR: 0.52, 95\% CI: 0.29-0.91, $\mathrm{P}=0.041$, Figure 3C). For patients with BMs at baseline, a significant association was uncovered between 

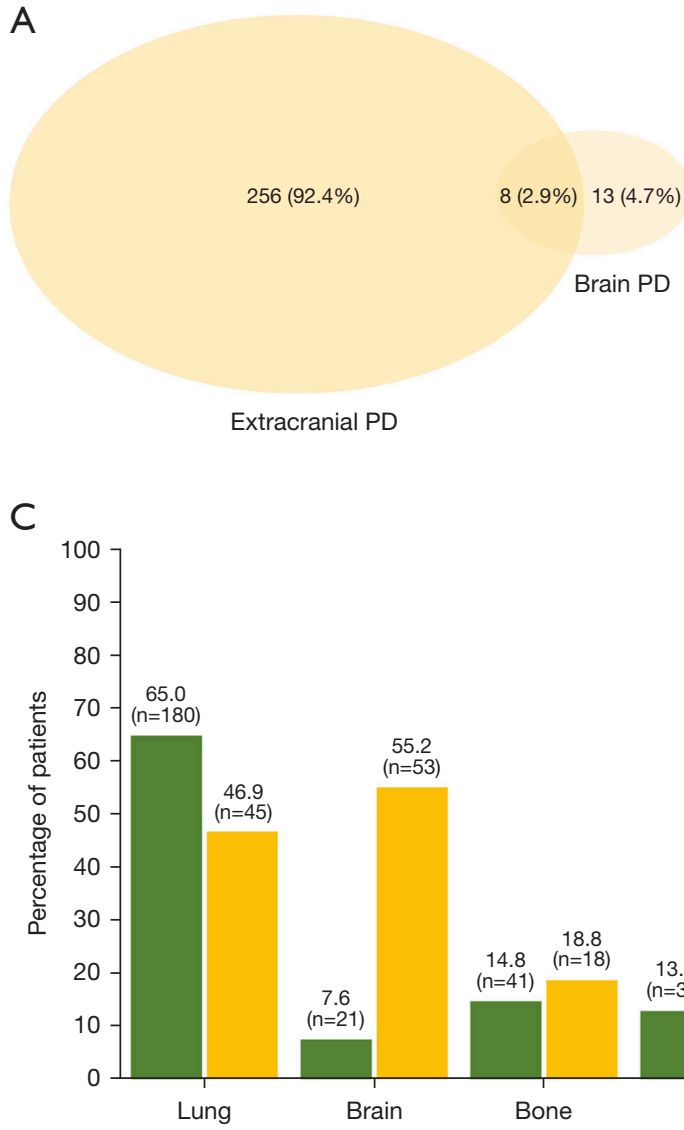
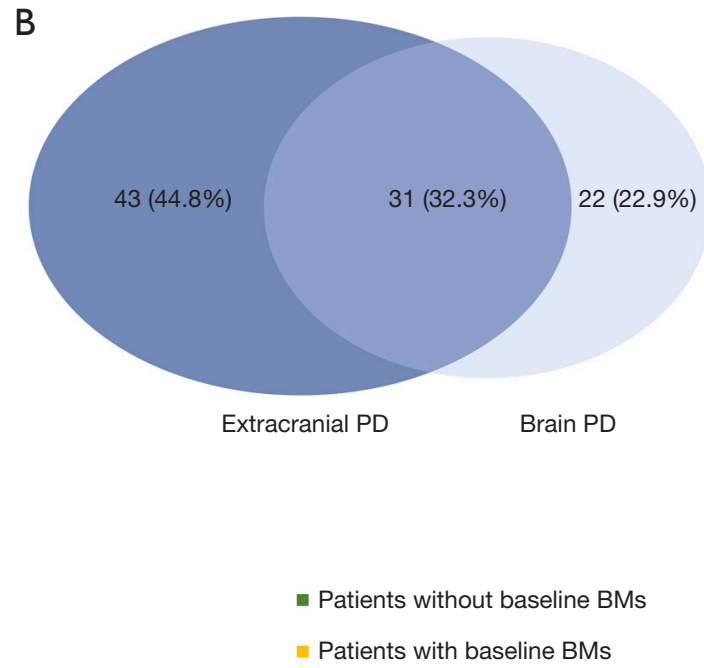

- Patients without baseline BMs

- Patients with baseline BMs

Figure 2 Patterns of initial disease progression analysis. (A) Venn diagram of patterns of initial progression in patients without baseline BMs; (B) Venn diagram of patterns of initial progression in patients with baseline BMs; (C) bar graph showing sites of initial progression on PD(L)1 inhibitor treatment. BMs, brain metastases; PD, progressive disease.

uCRT and a favorable survival outcome on univariate analysis (HR: 0.50, 95\% CI: $0.25-0.99, \mathrm{P}=0.045$ ), and this association retained borderline significance on multivariate analysis (HR $=0.55,95 \%$ CI: $0.28-1.10, \mathrm{P}=0.055$, Table S5).

The patients with baseline BMs were further subdivided into four groups according to the number of BMs (1-4 or $>4$ ) and receipt of uCRT (yes or no). The beneficial effects of uCRT in terms of iPFS, PFS, and OS were more prominent in patients with $1-4 \mathrm{BMs}(\mathrm{n}=83)$ (median iPFS, 20.6 vs. 4.7 months, HR: 0.47, 95\% CI: 0.26-0.87, $\mathrm{P}=0.034$, Figure $4 A$; median PFS, 4.8 vs. 3.0 months, HR: 0.55, 95\% CI: 0.33-0.90, $\mathrm{P}=0.035$, Figure $4 B$; median OS, 25.4 vs. 17.0 months, HR: $0.42,95 \%$ CI: $0.22-0.81, \mathrm{P}=0.024$, Figure $4 C)$ than in patients with $>4 \mathrm{BMs}(\mathrm{n}=27)$, among whom no significant benefit was indicated in terms of iPFS, PFS, or OS (median iPFS, 5.2 vs. 2.8 months, HR: 1.13, 95\% CI: 0.30-4.21, P=0.843, Figure 4A; median PFS, 1.8 vs. 1.8 months, HR: 0.84, 95\% CI: 0.36-1.95, $\mathrm{P}=0.686$, Figure 4B; median OS, 13.1 vs. 14.5 months, HR: 0.82, 95\% CI: $0.27-2.49, \mathrm{P}=0.730$, Figure $4 C$ ). Table $\mathrm{S} 6$ gives details on patients with 1-4 BMs. Among patients with 1-4 BMs, the proportion of cases with symptomatic BMs at the initiation of anti-PD-1/PD-L1 therapy was higher in the uCRT group than the non-uCRT group [33.3\% (6/18) vs. $10.8 \%$ (7/65), $\mathrm{P}=0.049$ ]. Univariate and multivariate analyses revealed that uCRT was independently associated with improved OS among patients with 1-4 BMs (univariate HR: $0.38,95 \%$ CI: $0.16-0.90, \mathrm{P}=0.028$; multivariate HR: 0.38, 95\% CI: 0.16-0.94, $\mathrm{P}=0.035$; Table 3).

\section{Discussion}

To date, numerous studies have been published focusing on the impact and timing of cranial RT in relation to anti- 
Table 2 Baseline characteristics of patients treated with versus without uCRT

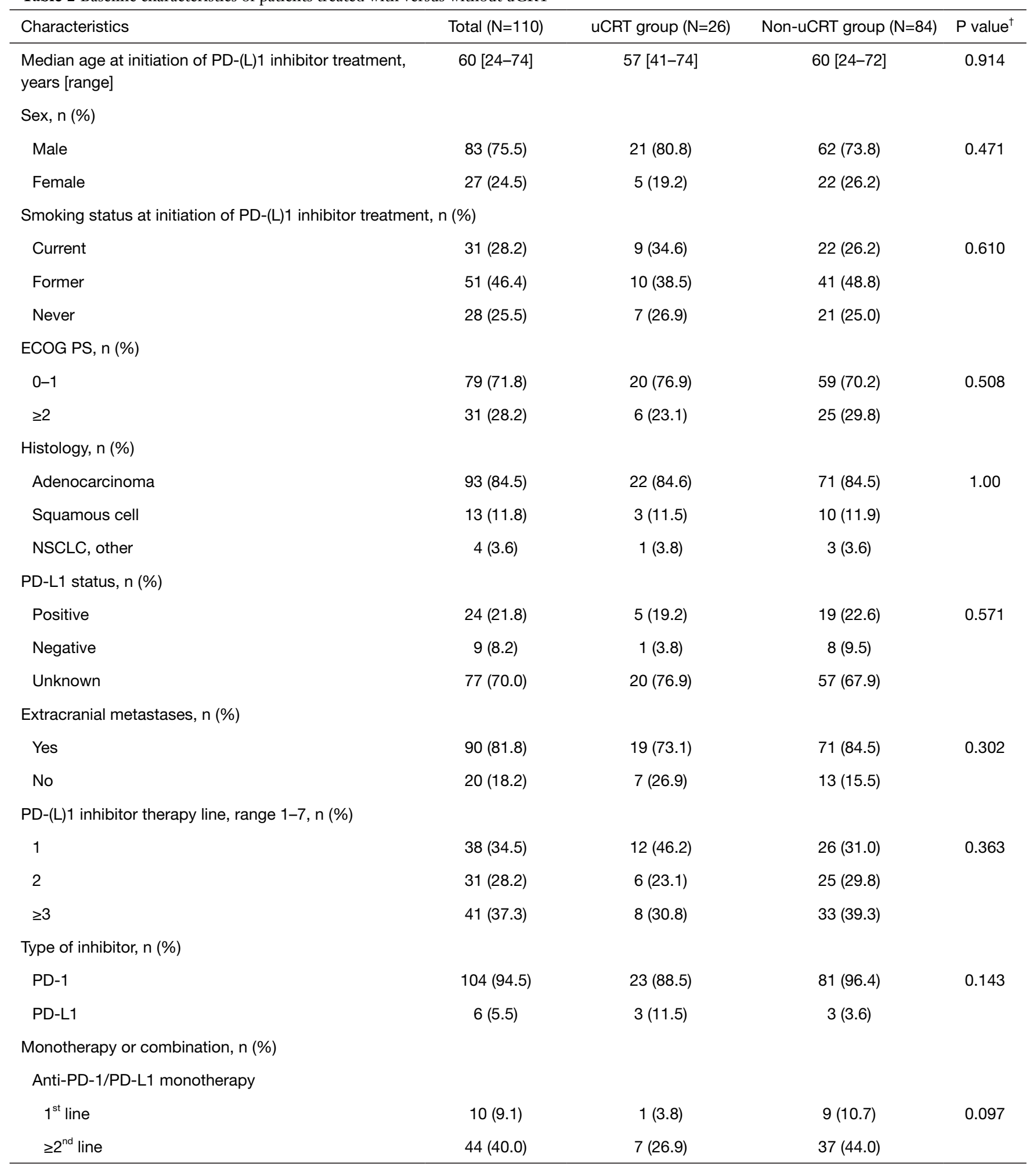

Table 2 (continued) 
Table 2 (continued)

\begin{tabular}{|c|c|c|c|c|}
\hline Characteristics & Total $(\mathrm{N}=110)$ & uCRT group $(\mathrm{N}=26)$ & Non-uCRT group $(\mathrm{N}=84)$ & $P$ value ${ }^{\dagger}$ \\
\hline $1^{\text {st }}$ line & $28(25.5)$ & $11(42.3)$ & $17(20.2)$ & \\
\hline$\geq 2^{\text {nd }}$ line & $28(25.5)$ & 7 (26.9) & $21(25.0)$ & \\
\hline \multicolumn{5}{|c|}{ No. of BMs, n (\%) } \\
\hline$>4$ & $27(24.5)$ & $8(30.8)$ & $19(22.6)$ & \\
\hline \multicolumn{5}{|c|}{ Diameter of largest BM, n (\%) } \\
\hline$<10 \mathrm{~mm}$ & $32(29.1)$ & $3(11.5)$ & $29(34.5)$ & 0.024 \\
\hline$\geq 10 \mathrm{~mm}$ & $78(70.9)$ & $23(88.5)$ & $55(65.5)$ & \\
\hline No & $88(80.0)$ & $17(65.4)$ & $71(84.5)$ & \\
\hline
\end{tabular}

${ }^{\dagger}$, patients treated with and without uCRT are compared; *, combination of anti-PD-1/PD-L1 therapy and chemotherapy or anti-PD-1/PD-L1 therapy and EGFR-TKIs. BMs, brain metastases; ECOG PS, Eastern Cooperative Oncology Group performance status; NSCLC, non-small cell lung cancer; PD-(L)1, programmed death receptor (ligand) 1; UCRT, upfront cranial radiotherapy.
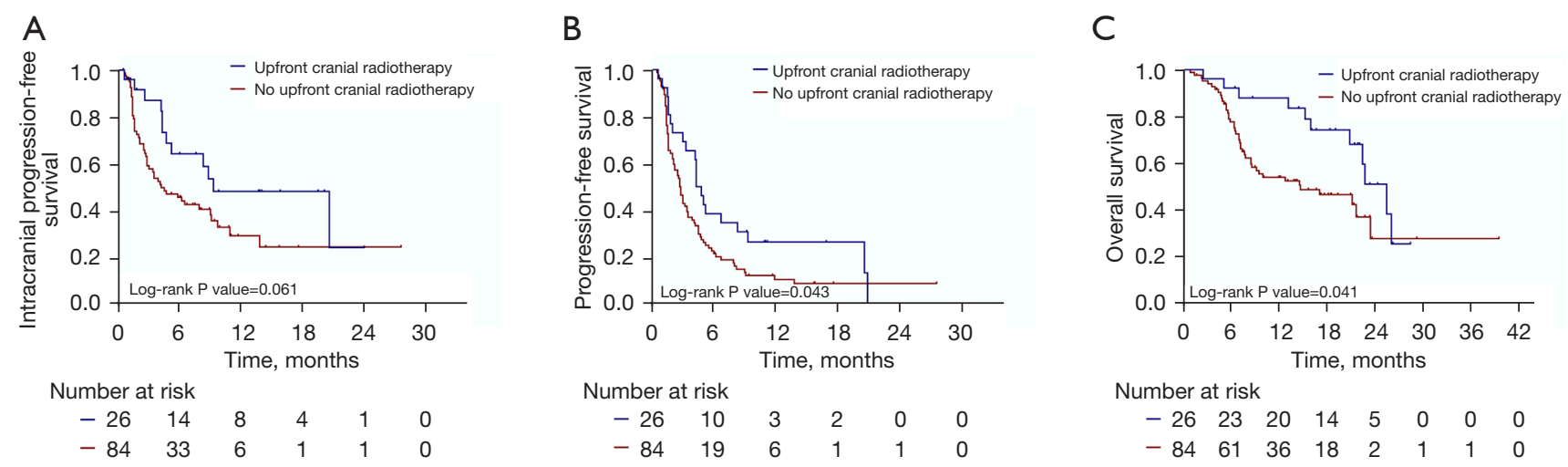

Figure 3 Kaplan-Meier curves for iPFS (A), PFS (B), and OS (C) according to the receipt of uCRT. iPFS, intracranial progression-free survival; OS, overall survival; PFS, progression-free survival.

PD-1/PD-L1 therapy for metastatic NSCLC (8,13-25). Combining cranial RT with PD-1/PD-L1 inhibitors has been demonstrated to provide better tumor control and longer survival than RT alone or exclusively systemic therapy, possibly owing to the favorable immunomodulatory effects of RT (7-9,13-25). For example, Shepard et al. reported that the median time to intracranial progression was 3.0 months in the concurrent-ICI cohort, and concurrent ICI and SRS led to an increased rate of complete response in the CNS for NSCLC-BM treated with SRS compared with SRS alone (50\% vs. 15.6\%, $\mathrm{P}=0.012)$ (17). Chen et al. retrospectively analyzed the impact of ICIs for patients with NSCLC, melanoma, and RCC with BMs treated with SRS/SRT (8); they reported a superior median OS in those receiving concurrent SRS/SRT and ICI relative to those who underwent SRS/SRT alone (24.7 vs. 12.9 months; $\mathrm{P}=0.002$ ). Furthermore, previous studies have reported extended OS and reduced rates of distant intracranial failure with concurrent PD-1/PD-L1 inhibitors and SRS/SRT compared to sequential therapy $(8,14)$. 

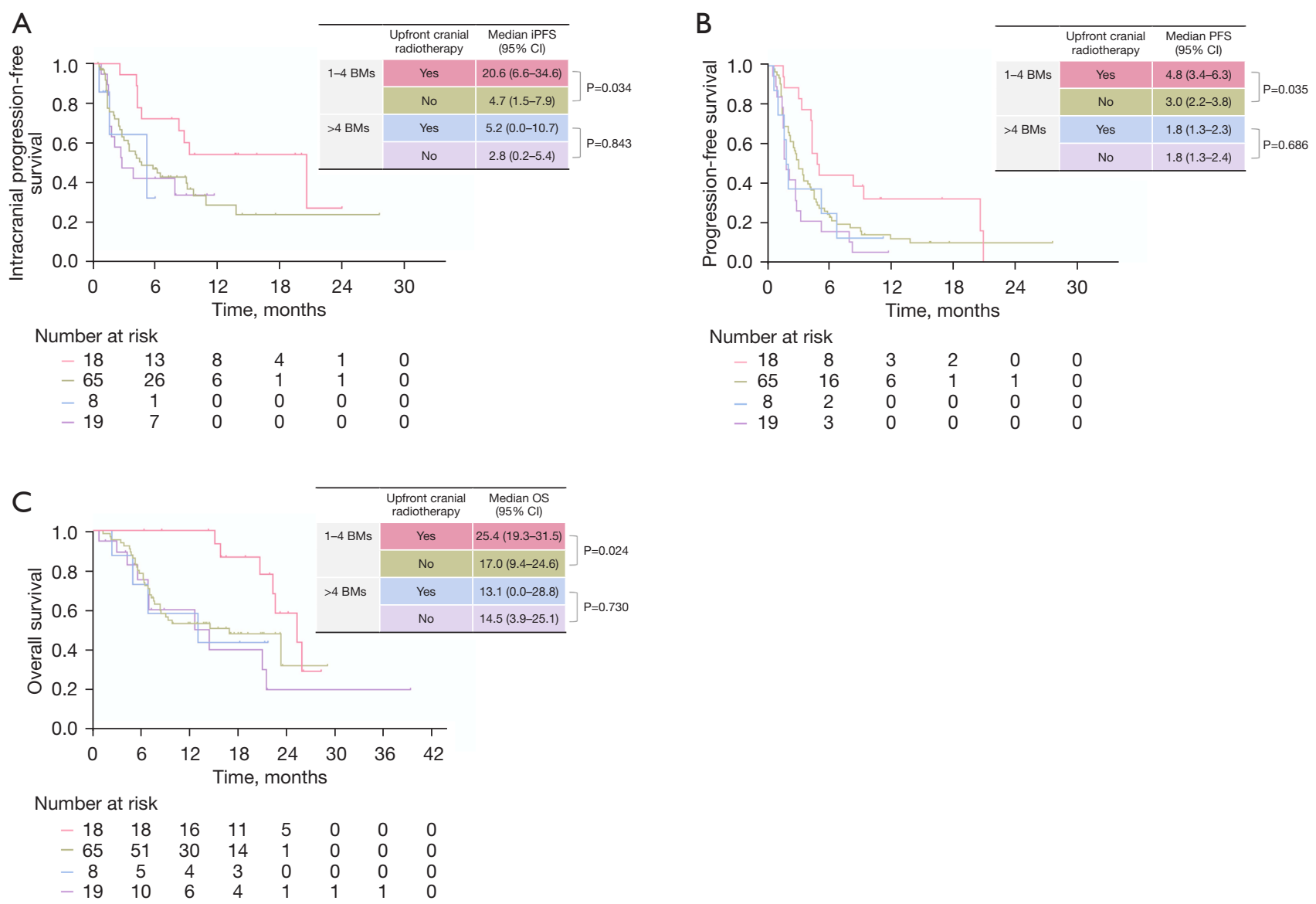

Figure 4 Kaplan-Meier curves for iPFS (A), PFS (B), and OS (C) according to the receipt of uCRT (yes vs. no) and the number of BMs (1-4 $v s$. >4). BMs, brain metastases; CI, confidence interval; iPFS, intracranial progression-free survival; OS, overall survival; PFS, progressionfree survival.

It is noteworthy that most published series are small, single-institution studies and have included heterogeneous groups of patients. Thus, there is still no consensus on the appropriate timing of cranial RT in conjunction to antiPD-1/PD-L1 therapy in the treatment of patients with brain-metastatic NSCLC. There are several unique features and strengths of the present study compared to previous investigations, e.g., its multi-institutional design, the unique insights it offers into the clinical value of uCRT via a detailed analysis of the patterns of initial and intracranial progression on PD-1/PD-L1 inhibitors and the impact of uCRT on clinical outcomes, focusing particularly on subgroups of patients stratified by the number of BMs.

Despite continuous and considerable efforts, the management of $\mathrm{BMs}$ remains a significant challenge in clinical practice. The recent wave of investigations establishing the clinical benefit of anti-PD-1/PD-L1 therapy for advanced NSCLC has also placed a spotlight on the role these immune checkpoint inhibitors can play for patients with BMs. Most studies demonstrated that BM originating from NSCLC was an immunosuppressive microenvironment, suggesting the intriguing possibility that immunotherapy may have a promising role in the treatment of NSCLC-BM (10). Accumulating evidence has shown encouraging results for the use of PD-1/PDL1 inhibitors in the treatment of brain-metastatic NSCLC (26-32). In our study of patients with metastatic NSCLC treated with PD-1/PD-L1 inhibitors, the median OS was similar between patients with and without BMs. Moreover, univariate and multivariate analyses revealed that the presence of BMs did not carry independent prognostic value for PFS or OS. These observations echo those of earlier studies, in which the presence of BMs at baseline was not identified to be an independent unfavorable prognostic 
Table 3 Univariate and multivariate Cox proportional-hazards model for OS in patients with 1-4 BMs

\begin{tabular}{|c|c|c|c|c|c|c|}
\hline Variables & \multicolumn{3}{|c|}{ Univariate analysis } & \multicolumn{3}{|c|}{ Multivariate analysis } \\
\hline Age ( $\leq 60$ vs. $>60$ years) & 1.01 & $0.74-1.38$ & 0.949 & - & - & - \\
\hline Sex (female vs. male) & 1.33 & $0.94-1.89$ & 0.111 & - & - & - \\
\hline Smoking status (never vs. current or former) & 1.00 & $0.69-1.46$ & 0.989 & - & - & - \\
\hline ECOG PS ( $\geq 2$ vs. $0-1)$ & 1.83 & $0.92-3.61$ & 0.083 & 1.31 & $0.63-2.72$ & 0.477 \\
\hline Extracranial metastases (yes vs. no) & 2.14 & $0.92-4.99$ & 0.077 & 1.65 & $0.67-4.04$ & 0.278 \\
\hline Lines of PD-(L)1 inhibitor treatment ( $\geq 2$ vs. 1 ) & 2.62 & $1.23-5.56$ & 0.012 & 2.07 & $0.93-4.61$ & 0.075 \\
\hline Treatment regimen (monotherapy vs combination therapy) & 1.75 & $0.92-3.32$ & 0.090 & 1.19 & $0.60-2.36$ & 0.622 \\
\hline Receipt of uCRT (yes vs. no) & 0.38 & $0.16-0.90$ & 0.028 & 0.38 & $0.16-0.94$ & 0.035 \\
\hline \multicolumn{7}{|l|}{ PD-L1 status } \\
\hline Positive & 0.62 & $0.17-2.28$ & 0.475 & - & - & - \\
\hline Unknown & 0.52 & $0.16-1.74$ & 0.287 & - & - & - \\
\hline Negative & Ref & - & - & - & - & - \\
\hline
\end{tabular}

BMs, brain metastases; Cl, confidence interval; ECOG PS, Eastern Cooperative Oncology Group performance status; HR, hazard ratio; OS, overall survival; PD-(L)1, programmed death receptor (ligand) 1; uCRT, upfront cranial radiotherapy.

factor for the OS of patients with PD-1/PD-L1 inhibitortreated NSCLC $(22,29,33)$. Therefore, when patients are treated with PD-1/PD-L1 inhibitors, the brain should not be considered an immune-privileged site, and in carefully selected patients with brain-metastatic NSCLC, anti-PD-1/ PD-L1 therapy may provide long-term survival benefit, which requires confirmation in future clinical trials.

The action of PD-1/PD-L1 inhibitors in the CNS can be owing to a dual mechanism: PD-1 blockade of T cells can occur in the systemic circulation before the cells penetrate the blood-brain barrier (BBB) and PD-1/PD-L1 inhibitors themselves can penetrate the $\mathrm{BBB}$ and have a direct intracranial antitumor effect. Recently, pembrolizumab CSF levels have been measured in 10 patients with highgrade gliomas treated with intravenous administration of pembrolizumab and intracranial administration of chimeric antigen receptor T cells (2). Results of this study revealed steady-state pembrolizumab CSF concentrations, and in vitro tumor rechallenge assays and PD-1 T-cell blocking data demonstrated that CSF concentrations were sufficient for blocking PD-1 on endogenous and adoptively transferred $\mathrm{T}$ cells, suggesting the direct intracranial effect of PD-1/PD-L1 inhibitors in the CNS.

Despite the promising efficacy of PD-1/PD-L1 inhibitors, the development of drug resistance is inevitable in most patients. In our research, the brain was the first site of progression more frequently in patients with baseline $\mathrm{BMs}$ than in those without $(55.2 \%$ vs. $7.6 \% ; \mathrm{P}<0.001)$. Among patients without baseline BMs, the 1-year actuarial rate of developing BMs was $10.0 \%$ and having a primary tumor $>2 \mathrm{~cm}$ and more than 2 organs involved in metastatic disease increased the likelihood of developing BMs. In most patients without initial CNS involvement who eventually suffered intracranial recurrence, extracranial recurrence preceded intracranial recurrence. Therefore, it is possible that distant metastases to the brain may result from systemic reseeding of resistant tumor clones which lack particular tumor antigens or antigen presentation, especially given that most patients in our study continued anti-PD-1/PDL1 therapy after initial recurrence. Among the patients with baseline BMs who progressed, the brain was the first site of progression in $55.2 \%$ of patients. Moreover, intracranial progression on PD-1/PD-L1 inhibitors occurred predominately at the original tumor sites in the brain. 
These observations suggest the intriguing possibility that uCRT before disease progression could carry the potential advantage of prolonging time to progression and improving overall outcomes, without even considering the profound immunomodulatory effects of RT and its potential synergy with anti-PD-1/PD-L1 immunotherapy.

To test this hypothesis, we classified patients with brainmetastatic NSCLC into two groups according to the receipt of uCRT. Our data showed that patients treated with uCRT had numerically longer iPFS and significantly improved PFS and OS compared to those who did not receive uCRT, which suggests a potential synergistic effect of $\mathrm{uCRT}$ in combination with anti-PD-1/PD-L1 therapy. It is noteworthy that in the uCRT group, heterogeneity in the modality of uCRT (WBRT or SRT/SRS) and the influence of the number of BMs on treatment selection can be a cause of concern; therefore, to mitigate this concern, we further subdivided patients with BMs into four groups according to the number of BMs and receipt of uCRT. The advantages of using uCRT in terms of iPFS, PFS, and OS were more prominent in patients with 1-4 BMs. Our findings suggest that upfront SRT/SRS may provide survival benefit for selected patients with a limited number of BMs (i.e., 1-4 BMs).

We chose 4 as the cutoff value between limited BMs and multiple BMs in this study for several reasons. First, WBRT is usually offered to patients with multiple BMs, while a maximum of 4 BMs was typically treated with SRS. To limited the selection bias associated with the influence of BM number on RT modality selection, patients with BMs were stratified into 1-4 BMs and >4 BMs groups. Second, the clinical value of cranial RT in conjunction with EGFR-TKIs or chemotherapy for NSCLC patients with 1-4 or $>4$ BMs has been widely investigated (34-36), while the role of cranial RT in patients stratified by the number of BMs remain a largely unexplored field in the era of immunotherapy. It is noteworthy that as the indications for SRT/SRS continue to expand, an increasing number of patients with multiple ( $>4)$ BMs could be considered for SRT/SRS instead of WBRT. More studies on the role of upfront SRT/SRS in the management of patients with multiple BMs treated with PD-1/PD-L1 inhibitors are needed.

There are a few limitations to the present study that should be acknowledged. First, the retrospective nature of this study makes it prone to confounding. For instance, the decision to incorporate $\mathrm{uCRT}$ into the treatment of patients with brain-metastatic NSCLC receiving PD-1/PD-L1 inhibitors may have been influenced by multiple factors, such as age, ECOG PS, the burden of intracranial disease, and clinical presentation (symptomatic vs. asymptomatic). In turn, these confounding factors could have influenced the outcome comparisons between the groups. Furthermore, we did not report on neurological adverse events due to the lack of clinical documentation. Previous literature suggests that the short-term safety profile of combined PD-1 pathway inhibition and cranial RT appeared to be acceptable, with no significant increase in RT- or immune-related adverse events observed in relation to this combination treatment $(8,13,14,37)$. Nevertheless, caution is warranted, as some reports have raised the concern that PD-1/PDL1 inhibitors could potentially exacerbate radiation-related necrosis (38-40), and the toxicities of this combination treatment, especially regarding long-term neurological and cognitive sequelae, still need to be established.

\section{Conclusions}

The presence of BMs at baseline was not associated with a survival difference in patients with metastatic NSCLC treated with PD-1/PD-L1 inhibitors. Most patients with PD-1/PD-L1 inhibitor-treated brain-metastatic NSCLC developed progressive intracranial disease at the original metastatic sites in the brain. Patients who received uCRT exhibited a longer median OS than those with BMs who did not receive uCRT; this survival advantage was more prominent in patients with 1-4 BMs. The findings obtained from the present study offer insight into and generate hypotheses related to the treatment of brain-metastatic NSCLC, which merit further investigation.

\section{Acknowledgments}

The authors appreciate the academic support from AME Lung Cancer Collaborative Group.

Funding: This work was supported by the Chinese Society of Clinical Oncology (Grant Nos. Y-BMS2019082, Y-MSD20200147).

\section{Footnote}

Reporting Checklist: The authors have completed the STROBE reporting checklist. Available at https://tlcr. amegroups.com/article/view/10.21037/tlcr-22-54/rc

Data Sharing Statement: Available at https://tlcr.amegroups. 
com/article/view/10.21037/tlcr-22-54/dss

Conflicts of Interest: All authors have completed the ICMJE uniform disclosure form (available at https://tlcr.amegroups. com/article/view/10.21037/tlcr-22-54/coif). Dr. KWM receives Research Grant for clinical trials from AstraZeneca and Novartis, Education Grant from Pfizer and Varian; gets Travel support for a research meeting from AstraZeneca; and sits on Board of Directors of Global Access to Cancer Care Foundation. The other authors have no conflicts of interest to declare.

Ethical Statement: The authors are accountable for all aspects of the work in ensuring that questions related to the accuracy or integrity of any part of the work are appropriately investigated and resolved. The study was conducted in accordance with the Declaration of Helsinki (as revised in 2013). The study was approved by the Institutional Review Board of Fudan University Shanghai Cancer Center (No. 2012228-3), Ethics Committee of Zhongshan Hospital, Fudan University (No. B2021-125R), and Ethics Committee of the Tongji Medical College of Huazhong University of Science and Technology (No. TJIRB20200731), and individual consent for this retrospective analysis was waived.

Open Access Statement: This is an Open Access article distributed in accordance with the Creative Commons Attribution-NonCommercial-NoDerivs 4.0 International License (CC BY-NC-ND 4.0), which permits the noncommercial replication and distribution of the article with the strict proviso that no changes or edits are made and the original work is properly cited (including links to both the formal publication through the relevant DOI and the license). See: https://creativecommons.org/licenses/by-nc-nd/4.0/.

\section{References}

1. van Bussel MTJ, Beijnen JH, Brandsma D. Intracranial antitumor responses of nivolumab and ipilimumab: a pharmacodynamic and pharmacokinetic perspective, a scoping systematic review. BMC Cancer 2019;19:519.

2. Portnow J, Wang D, Blanchard MS, et al. Systemic Anti-PD-1 Immunotherapy Results in PD-1 Blockade on T Cells in the Cerebrospinal Fluid. JAMA Oncol 2020;6:1947-51.

3. Borghaei H, Paz-Ares L, Horn L, et al. Nivolumab versus Docetaxel in Advanced Nonsquamous Non-Small-Cell
Lung Cancer. N Engl J Med 2015;373:1627-39.

4. Brahmer J, Reckamp KL, Baas P, et al. Nivolumab versus Docetaxel in Advanced Squamous-Cell Non-Small-Cell Lung Cancer. N Engl J Med 2015;373:123-35.

5. Rittmeyer A, Barlesi F, Waterkamp D, et al. Atezolizumab versus docetaxel in patients with previously treated non-small-cell lung cancer (OAK): a phase 3, openlabel, multicentre randomised controlled trial. Lancet 2017;389:255-65.

6. Socinski MA, Jotte RM, Cappuzzo F, et al. Atezolizumab for First-Line Treatment of Metastatic Nonsquamous NSCLC. N Engl J Med 2018;378:2288-301.

7. Shaverdian N, Lisberg AE, Bornazyan K, et al. Previous radiotherapy and the clinical activity and toxicity of pembrolizumab in the treatment of non-small-cell lung cancer: a secondary analysis of the KEYNOTE-001 phase 1 trial. Lancet Oncol 2017;18:895-903.

8. Chen L, Douglass J, Kleinberg L, et al. Concurrent Immune Checkpoint Inhibitors and Stereotactic Radiosurgery for Brain Metastases in Non-Small Cell Lung Cancer, Melanoma, and Renal Cell Carcinoma. Int J Radiat Oncol Biol Phys 2018;100:916-25.

9. Knisely JP, Yu JB, Flanigan J, et al. Radiosurgery for melanoma brain metastases in the ipilimumab era and the possibility of longer survival. J Neurosurg 2012;117:227-33.

10. Xiao G, Liu Z, Gao X, et al. Immune checkpoint inhibitors for brain metastases in non-small-cell lung cancer: from rationale to clinical application. Immunotherapy 2021;13:1031-51.

11. Turkaj A, Morelli AM, Vavalà T, et al. Management of Leptomeningeal Metastases in Non-oncogene Addicted Non-small Cell Lung Cancer. Front Oncol 2018;8:278.

12. Huang Q, Zhang H, Hai J, et al. Impact of PD-L1 expression, driver mutations and clinical characteristics on survival after anti-PD-1/PD-L1 immunotherapy versus chemotherapy in non-small-cell lung cancer: A meta-analysis of randomized trials. Oncoimmunology 2018;7:e1396403.

13. Ahmed KA, Kim S, Arrington J, et al. Outcomes targeting the PD-1/PD-L1 axis in conjunction with stereotactic radiation for patients with non-small cell lung cancer brain metastases. J Neurooncol 2017;133:331-8.

14. Schapira E, Hubbeling H, Yeap BY, et al. Improved Overall Survival and Locoregional Disease Control With Concurrent PD-1 Pathway Inhibitors and Stereotactic Radiosurgery for Lung Cancer Patients With Brain Metastases. Int J Radiat Oncol Biol Phys 2018;101:624-9. 15. Singh C, Qian JM, Yu JB, et al. Local tumor response and 
survival outcomes after combined stereotactic radiosurgery and immunotherapy in non-small cell lung cancer with brain metastases. J Neurosurg 2019;132:512-7.

16. Crinò L, Bronte G, Bidoli P, et al. Nivolumab and brain metastases in patients with advanced non-squamous nonsmall cell lung cancer. Lung Cancer 2019;129:35-40.

17. Shepard MJ, Xu Z, Donahue J, et al. Stereotactic radiosurgery with and without checkpoint inhibition for patients with metastatic non-small cell lung cancer to the brain: a matched cohort study. J Neurosurg 2019. [Epub ahead of print].

18. Lanier CM, Hughes R, Ahmed T, et al. Immunotherapy is associated with improved survival and decreased neurologic death after SRS for brain metastases from lung and melanoma primaries. Neurooncol Pract 2019;6:402-9.

19. Qian JM, Martin AM, Martin K, et al. Response rate and local recurrence after concurrent immune checkpoint therapy and radiotherapy for non-small cell lung cancer and melanoma brain metastases. Cancer 2020;126:5274-82.

20. Travis RL, Marcrom SR, Brown MH, et al. Control and Toxicity in Melanoma Versus Other Brain Metastases in Response to Combined Radiosurgery and PD-(L)1 Immune Checkpoint Inhibition. Adv Radiat Oncol 2020;6:100561.

21. Metro G, Gili A, Signorelli D, et al. Upfront pembrolizumab as an effective treatment start in patients with PD-L1 $\geq 50 \%$ non-oncogene addicted non-small cell lung cancer and asymptomatic brain metastases: an exploratory analysis. Clin Transl Oncol 2021;23:1818-26.

22. Sun L, Davis CW, Hwang WT, et al. Outcomes in Patients With Non-small-cell Lung Cancer With Brain Metastases Treated With Pembrolizumab-based Therapy. Clin Lung Cancer 2021;22:58-66.e3.

23. Lau SCM, Poletes C, Le LW, et al. Durability of CNS disease control in NSCLC patients with brain metastases treated with immune checkpoint inhibitors plus cranial radiotherapy. Lung Cancer 2021;156:76-81.

24. Rounis K, Skribek M, Makrakis D, et al. Correlation of Clinical Parameters with Intracranial Outcome in NonSmall Cell Lung Cancer Patients with Brain Metastases Treated with Pd-1/Pd-L1 Inhibitors as Monotherapy. Cancers (Basel) 2021;13:1562.

25. Goldberg SB, Schalper KA, Gettinger SN, et al. Pembrolizumab for management of patients with NSCLC and brain metastases: long-term results and biomarker analysis from a non-randomised, open-label, phase 2 trial. Lancet Oncol 2020;21:655-63.
26. Cortinovis D, Chiari R, Catino A, et al. Italian Cohort of the Nivolumab EAP in Squamous NSCLC: Efficacy and Safety in Patients With CNS Metastases. Anticancer Res 2019;39:4265-71.

27. Zhang G, Cheng R, Wang H, et al. Comparable outcomes of nivolumab in patients with advanced NSCLC presenting with or without brain metastases: a retrospective cohort study. Cancer Immunol Immunother 2020;69:399-405.

28. Grossi F, Genova C, Crinò L, et al. Real-life results from the overall population and key subgroups within the Italian cohort of nivolumab expanded access program in non-squamous non-small cell lung cancer. Eur J Cancer 2019;123:72-80.

29. Hendriks LEL, Henon C, Auclin E, et al. Outcome of Patients with Non-Small Cell Lung Cancer and Brain Metastases Treated with Checkpoint Inhibitors. J Thorac Oncol 2019;14:1244-54.

30. Gauvain C, Vauléon E, Chouaid C, et al. Intracerebral efficacy and tolerance of nivolumab in non-small-cell lung cancer patients with brain metastases. Lung Cancer 2018;116:62-6.

31. Gadgeel SM, Lukas RV, Goldschmidt J, et al. Atezolizumab in patients with advanced non-small cell lung cancer and history of asymptomatic, treated brain metastases: Exploratory analyses of the phase III OAK study. Lung Cancer 2019;128:105-12.

32. Dudnik E, Yust-Katz S, Nechushtan H, et al. Intracranial response to nivolumab in NSCLC patients with untreated or progressing CNS metastases. Lung Cancer 2016;98:114-7.

33. Qiao M, Zhou F, Hou L, et al. Efficacy of immunecheckpoint inhibitors in advanced non-small cell lung cancer patients with different metastases. Ann Transl Med 2021;9:34.

34. Miyawaki E, Kenmotsu H, Mori K, et al. Optimal Sequence of Local and EGFR-TKI Therapy for EGFRMutant Non-Small Cell Lung Cancer With Brain Metastases Stratified by Number of Brain Metastases. Int J Radiat Oncol Biol Phys 2019;104:604-13.

35. Lim SH, Lee JY, Lee MY, et al. A randomized phase III trial of stereotactic radiosurgery (SRS) versus observation for patients with asymptomatic cerebral oligo-metastases in non-small-cell lung cancer. Ann Oncol 2015;26:762-8.

36. Wang C, Lu X, Lyu Z, et al. Comparison of up-front radiotherapy and TKI with TKI alone for NSCLC with brain metastases and EGFR mutation: A meta-analysis. Lung Cancer 2018;122:94-9.

37. Hubbeling HG, Schapira EF, Horick NK, et al. Safety 
of Combined PD-1 Pathway Inhibition and Intracranial Radiation Therapy in Non-Small Cell Lung Cancer. J Thorac Oncol 2018;13:550-8.

38. Kluger HM, Chiang V, Mahajan A, et al. Long-Term Survival of Patients With Melanoma With Active Brain Metastases Treated With Pembrolizumab on a Phase II Trial. J Clin Oncol 2019;37:52-60.

39. Alomari AK, Cohen J, Vortmeyer AO, et al. Possible

Cite this article as: Guo T, Chu L, Chu X, Yang X, Li Y, Zhou Y, Xu D, Zhang J, Wang S, Hu J, Chu Q, Moran T, Cho WCS, Merrell KW, Rizzo S, Liu Y, Ni J, Zhu Z. Brain metastases, patterns of intracranial progression, and the clinical value of upfront cranial radiotherapy in patients with metastatic non-small cell lung cancer treated with PD-1/PD-L1 inhibitors. Transl Lung Cancer Res 2022;11(2):173-187. doi: 10.21037/ tlcr-22-54
Interaction of Anti-PD-1 Therapy with the Effects of Radiosurgery on Brain Metastases. Cancer Immunol Res 2016;4:481-7.

40. Colaco RJ, Martin P, Kluger HM, et al. Does immunotherapy increase the rate of radiation necrosis after radiosurgical treatment of brain metastases? J Neurosurg 2016;125:17-23. 


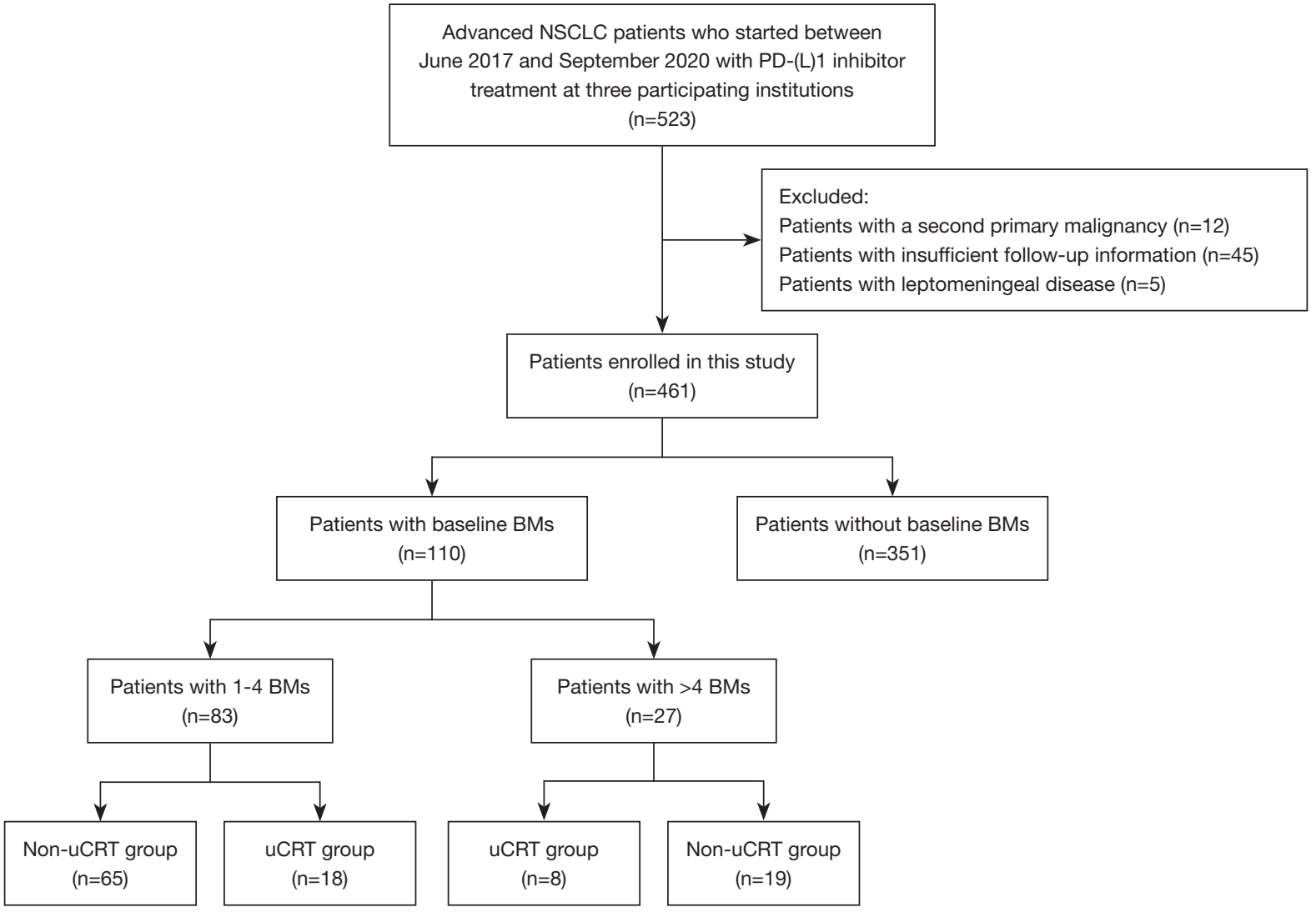

Figure S1 Flowchart of patient enrollment. BMs, brain metastases; NSCLC, non-small cell lung cancer; PD-(L)1, programmed death receptor (ligand) 1; uCRT, upfront cranial radiotherapy. 
Table S1 Brain metastases and treatment characteristics of 110 patients with BMs at baseline

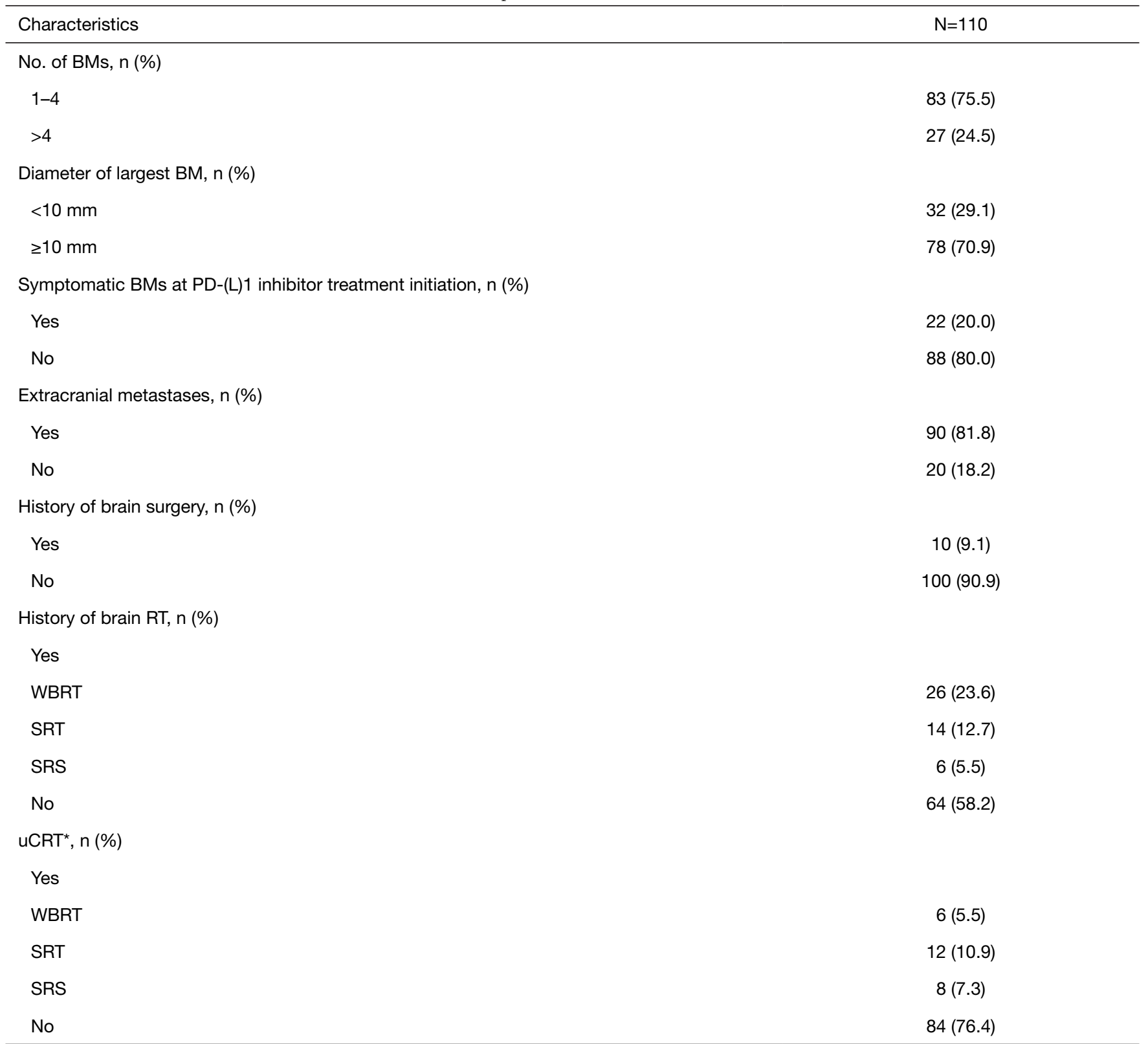

*, uCRT was defined as the receipt of cranial irradiation at any point between the initiation of anti-PD-1/PD-L1 therapy and the first subsequent progression event. BMs, brain metastases; PD-(L)1, programmed death receptor (ligand) 1; RT, radiotherapy; uCRT, upfront cranial radiotherapy; SRS, stereotactic radiosurgery; SRT, stereotactic radiotherapy; WBRT, whole-brain radiation therapy. 
Table S2 Cox proportional-hazards analyses for PFS in the whole study population

\begin{tabular}{|c|c|c|c|c|c|c|}
\hline Variables & \multicolumn{3}{|c|}{ Univariate analysis } & \multicolumn{3}{|c|}{ Multivariate analysis } \\
\hline Age ( $\leq 60$ vs. $>60$ years) & 0.93 & $0.76-1.14$ & 0.466 & & & \\
\hline Sex (male vs. female) & 1.38 & $1.08-1.77$ & 0.011 & 1.12 & $0.87-1.45$ & 0.371 \\
\hline Smoking status (never vs. current or former) & 1.10 & $0.98-1.23$ & 0.111 & & & \\
\hline ECOG PS ( $\geq 2$ vs. $0-1)$ & 1.92 & $1.52-2.42$ & $<0.001$ & 1.49 & $1.16-1.91$ & 0.002 \\
\hline No. of organs harboring metastases (>2 vs. $\leq 2$ ) & 2.60 & $2.06-3.29$ & $<0.001$ & 1.94 & $1.48-2.53$ & $<0.001$ \\
\hline Lines of PD-(L)1 inhibitor treatment ( $\geq 2$ vs. 1 ) & 1.71 & $1.39-2.11$ & $<0.001$ & 1.53 & $1.22-1.91$ & $<0.001$ \\
\hline Treatment regimen (monotherapy vs. combination therapy) & 1.07 & $0.87-1.32$ & 0.514 & & & \\
\hline \multicolumn{7}{|l|}{ PD-L1 status } \\
\hline Positive & 0.53 & $0.34-0.83$ & 0.005 & 0.66 & $0.44-0.99$ & 0.046 \\
\hline Unknown & 0.65 & $0.44-0.97$ & 0.033 & 0.67 & $0.42-1.06$ & 0.085 \\
\hline Negative & Ref & - & - & Ref & - & - \\
\hline
\end{tabular}

BMs, brain metastases; Cl, confidence interval; ECOG PS, Eastern Cooperative Oncology Group performance status; HR, hazard ratio; PD-(L)1, programmed death receptor (ligand) 1; PFS, progression-free survival.

Table S3 Cox proportional-hazards analyses for OS in the whole study population

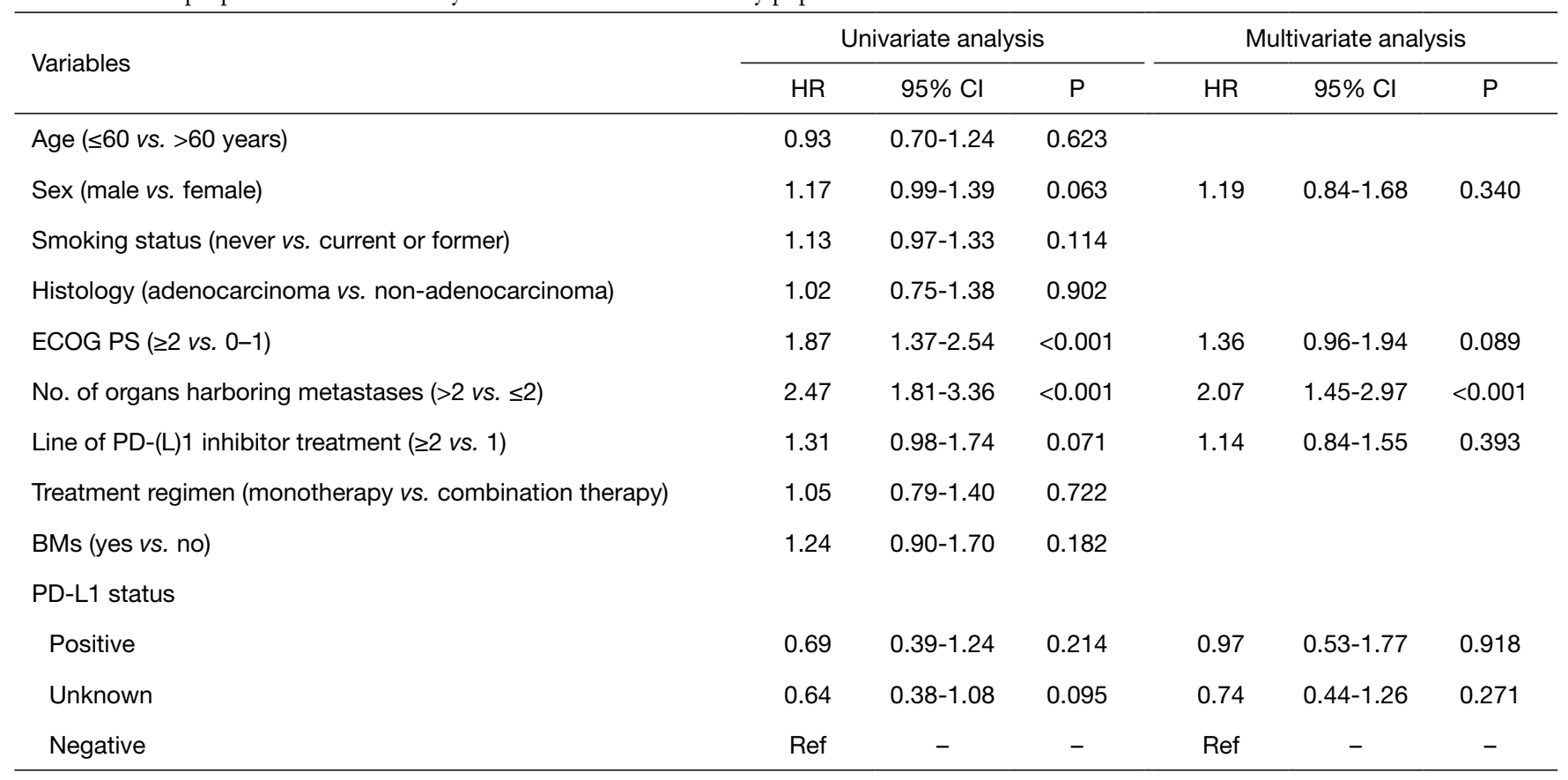

BM, brain metastases; Cl, confidence interval; ECOG PS, Eastern Cooperative Oncology Group performance status; HR, hazard ratio; OS, overall survival; PD-(L)1, programmed death receptor (ligand) 1. 
Table S4 Predictors of the development of BMs for patients without baseline BMs who were treated with anti-PD-(L)1 therapy

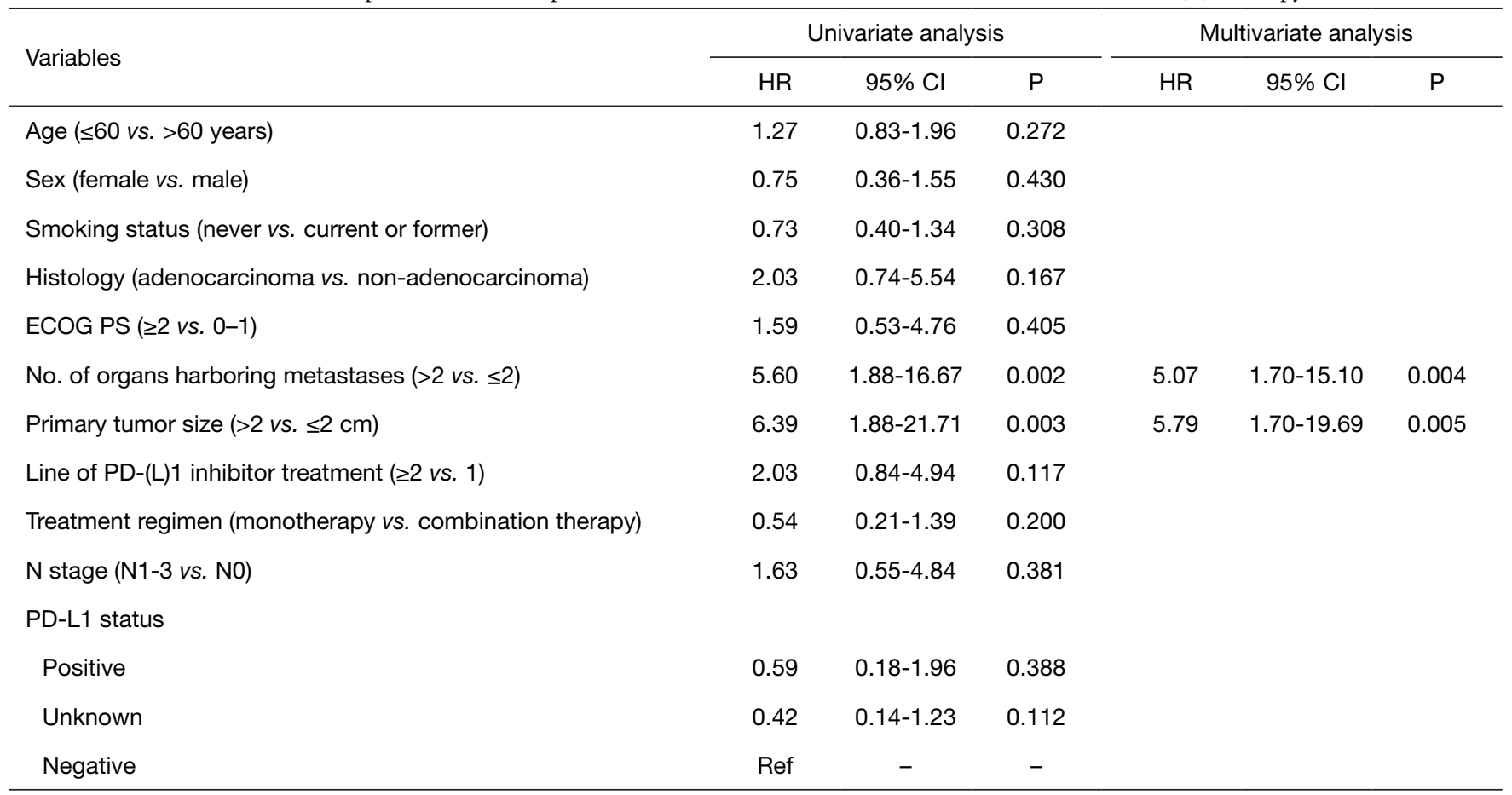

BMs, brain metastases; Cl, confidence interval; ECOG PS, Eastern Cooperative Oncology Group performance status; HR, hazard ratio; PD-(L)1, programmed death receptor (ligand) 1.

Table S5 Univariate and multivariate Cox proportional-hazards model for OS in patients with BMs

\begin{tabular}{|c|c|c|c|c|c|c|}
\hline Variables & \multicolumn{3}{|c|}{ Univariate analysis } & \multicolumn{3}{|c|}{ Multivariate analysis } \\
\hline Age ( $\leq 60$ vs. $>60$ years) & 0.85 & $0.50-1.46$ & 0.563 & & & \\
\hline Sex (female vs. male) & 1.38 & $1.03-1.85$ & 0.030 & 1.66 & $0.91-3.04$ & 0.030 \\
\hline Smoking status (never vs. current or former) & 1.00 & $0.74-1.36$ & 0.995 & & & \\
\hline ECOG PS ( $\geq 2$ vs. $0-1)$ & 1.36 & $0.74-2.49$ & 0.325 & & & \\
\hline Extracranial metastases (yes vs. no) & 2.01 & $0.93-4.32$ & 0.075 & 1.68 & $0.76-3.71$ & 0.098 \\
\hline Lines of PD-(L)1 inhibitor treatment ( $\geq 2$ vs. 1 ) & 1.77 & $0.97-3.23$ & 0.065 & 1.30 & $0.69-2.47$ & 0.153 \\
\hline Treatment regimen (monotherapy vs. combination therapy) & 1.21 & $0.71-2.08$ & 0.478 & & & \\
\hline Receipt of uCRT (yes vs. no) & 0.50 & $0.25-0.99$ & 0.045 & 0.55 & $0.28-1.10$ & 0.055 \\
\hline \multicolumn{7}{|l|}{ PD-L1 status } \\
\hline Positive & 0.65 & $0.21-2.06$ & 0.467 & & & \\
\hline Unknown & 0.67 & $0.24-1.89$ & 0.448 & & & \\
\hline Negative & Ref & - & - & & & \\
\hline
\end{tabular}

BMs, brain metastases; Cl, confidence interval; ECOG PS, Eastern Cooperative Oncology Group performance status; HR, hazard ratio; OS, overall survival; PD-(L)1, programmed death receptor (ligand) 1; uCRT, upfront cranial radiotherapy. 
Table S6 Baseline characteristics of patients with 1-4 BMs, stratified according to the receipt of uCRT

\begin{tabular}{|c|c|c|c|c|}
\hline Characteristics & Total $(\mathrm{N}=83)$ & uCRT group $(\mathrm{N}=18)$ & Non-uCRT group $(\mathrm{N}=65)$ & $P$ value ${ }^{\dagger}$ \\
\hline Median age at initiation of PD-(L)1 inhibitor treatment, years (range) & $60(34-74)$ & $60(41-74)$ & $61(34-72)$ & 0.891 \\
\hline \multicolumn{5}{|l|}{ Sex, $n(\%)$} \\
\hline Male & $65(78.3)$ & $15(83.3)$ & $50(76.9)$ & \multirow[t]{2}{*}{0.794} \\
\hline Female & $18(21.7)$ & $3(16.7)$ & $15(23.1)$ & \\
\hline \multicolumn{5}{|l|}{ Smoking status at PD-(L)1 inhibitor treatment initiation, $\mathrm{n}(\%)$} \\
\hline Current & $26(31.3)$ & $7(38.9)$ & $19(29.2)$ & \multirow[t]{3}{*}{0.491} \\
\hline Former & $40(48.2)$ & $9(50.0)$ & $31(47.7)$ & \\
\hline Never & $17(20.5)$ & $2(11.1)$ & $15(23.1)$ & \\
\hline \multicolumn{5}{|l|}{ ECOG PS, n (\%) } \\
\hline $0-1$ & $61(73.5)$ & $13(72.2)$ & $48(73.8)$ & \multirow[t]{2}{*}{1.00} \\
\hline$\geq 2$ & $22(26.5)$ & $5(27.8)$ & $17(26.2)$ & \\
\hline \multicolumn{5}{|l|}{ Histology, n (\%) } \\
\hline Adenocarcinoma & $71(85.5)$ & $15(83.3)$ & $56(86.2)$ & \multirow[t]{3}{*}{0.473} \\
\hline Squamous cell & $9(10.8)$ & $3(16.7)$ & $6(9.2)$ & \\
\hline NSCLC, other & $3(3.6)$ & $0(0.0)$ & $3(4.6)$ & \\
\hline \multicolumn{5}{|l|}{ PD-L1 status, n (\%) } \\
\hline Positive & $22(26.5)$ & $4(22.2)$ & $18(27.7)$ & \multirow[t]{3}{*}{0.608} \\
\hline Negative & $5(6.0)$ & $0(0.0)$ & $5(7.7)$ & \\
\hline Unknown & $56(67.5)$ & $14(77.8)$ & $42(64.6)$ & \\
\hline \multicolumn{5}{|l|}{ Extracranial metastases, $\mathrm{n}(\%)$} \\
\hline Yes & $64(77.1)$ & $12(66.7)$ & $52(80.0)$ & \multirow[t]{2}{*}{0.382} \\
\hline No & $19(22.9)$ & $6(33.3)$ & $13(20.0)$ & \\
\hline \multicolumn{5}{|l|}{ PD-(L)1 inhibitor therapy line, range 1-7 } \\
\hline 1 & $31(37.3)$ & $9(50.0)$ & $22(33.8)$ & \multirow[t]{3}{*}{0.392} \\
\hline 2 & $22(26.5)$ & $3(16.7)$ & $19(29.2)$ & \\
\hline$\geq 3$ & $30(36.1)$ & $6(33.3)$ & $24(36.9)$ & \\
\hline \multicolumn{5}{|l|}{ Type of inhibitor } \\
\hline PD-1 & $77(92.8)$ & $15(83.3)$ & $62(95.4)$ & \multirow[t]{2}{*}{0.113} \\
\hline PD-L1 & $6(7.2)$ & $3(16.7)$ & $3(4.6)$ & \\
\hline \multicolumn{5}{|l|}{ Monotherapy or combination } \\
\hline \multicolumn{5}{|l|}{ Anti-PD-1/PD-L1 monotherapy } \\
\hline $1^{\text {st }}$ line & $9(10.8)$ & $1(5.6)$ & $8(12.3)$ & \multirow[t]{5}{*}{0.235} \\
\hline$\geq 2^{\text {nd }}$ line & $35(42.2)$ & $5(27.8)$ & 30 (46.2) & \\
\hline \multicolumn{4}{|l|}{ Combination* } & \\
\hline $1^{\text {st }}$ line & $22(26.5)$ & $8(44.4)$ & $14(21.5)$ & \\
\hline$\geq 2^{\text {nd }}$ line & $17(20.5)$ & $4(22.2)$ & $13(20.0)$ & \\
\hline Diameter of largest BM, $\mathrm{n}(\%)$ & & & & \\
\hline$<10 \mathrm{~mm}$ & $27(32.5)$ & $3(16.7)$ & $24(36.9)$ & \multirow[t]{2}{*}{0.105} \\
\hline$\geq 10 \mathrm{~mm}$ & $56(67.5)$ & $15(83.3)$ & $41(63.1)$ & \\
\hline \multicolumn{5}{|l|}{ Symptomatic BMs at PD-(L)1 inhibitor treatment initiation, $\mathrm{n}(\%)$} \\
\hline Yes & $13(15.7)$ & $6(33.3)$ & $7(10.8)$ & \multirow[t]{2}{*}{0.049} \\
\hline No & 70 (84.3) & $12(66.7)$ & 58 (89.2) & \\
\hline
\end{tabular}

${ }^{\dagger}$, patients treated with and without upfront cranial radiotherapy are compared; *, combination of anti-PD-1/PD-L1 therapy and chemotherapy or anti-PD-1/PD-L1 therapy and EGFR-TKIs. BMs, brain metastases; ECOG PS, Eastern Cooperative Oncology Group performance status; NSCLC, non-small cell lung cancer; PD-(L)1, programmed death receptor (ligand) 1; uCRT, upfront cranial radiotherapy. 